\section{Prevalencia y caracterización del perfil de susceptibilidad antimicrobiana de Campylobacter jejuni y Campylobacter coli en niños con diarrea de la ciudad de Loja, Ecuador}

\author{
Rosa Simaluiza, Zorayda Toledo y Heriberto Fernández
}

\author{
Prevalence and antimicrobial behavior of \\ Campylobacter jejuni and Campylobacter coli in \\ children with diarrhea in Loja city, Ecuador
}

Introduction: Campylobacter is an important agent of diarrhea in humans. In Ecuador, the information on Campylobacter is scarce and there are not antecedents about antimicrobial susceptibility. Objective: To describe Campylobacter prevalence in children with diarrhea and their behavior against five antimicrobials in vitro. Method: We studied 253 children with diarrhea aging 7 months to 9 years who consulted for diarrhea in two hospitals in the city of Loja. Fecal samples were cultured and identification by tests by phenotypic tests and multiplex PCR. Susceptibility to 5 antibiotics was determined by the disc-diffusion method. Results: Campylobacter was found in $16(6.3 \%)$ children, being C. jejuni the most frequent one (5.1\%), followed by C. coli (1.2\%). All strains were susceptible to gentamicin and ampicillin/clavulanic acid, being found low resistance to ampicillin and erythromycin and high resistance to ciprofloxacin.

Key words: Campylobacter; diarrhea; children; Ecuador; antimicrobial resistance.

Palabras clave: Campylobacter; diarrea; niños; Ecuador; resistencia antimicrobiana.

\section{Introducción}

Las especies termotolerantes de Campylobacter (Campylobacter jejuni, Campylobacter coli, Campylobacter lari y más recientemente Campylobacter upsaliensis) han adquirido gran importancia en salud pública, especialmente como agentes causales de diarrea infecciosa en el ser humano ${ }^{1,2}$. De estas cuatro especies, $C$. jejuni es la más frecuentemente aislada, tanto en países en vías de desarrollo como en países industrializados. Campylobacter coli le sigue en frecuencia y, en Sudamérica, es responsable de cerca de $25 \%$ de los casos de diarrea atribuibles a especies

Universidad Técnica Particular de Loja, Loja, Ecuador. Departamento de Ciencias de la Salud, Sección Genética Humana, Microbiología y Bioquímica Clínica (RS, ST).

Universidad Austral de Chile. Valdivia, Chile. Instituto de Microbiología Clínica (HF).

Sin conflictos de interés.

Fuente de financiamiento: Este manuscrito fue financiado por la Universidad Técnica Particular de Loja, proyecto PROY-CCSAL_ 1077.

Recibido: 14 de septiembre de 2017 / Aceptado: 20 de marzo de 2018

\section{Correspondencia a:}

Heriberto Fernández

hfernand@uach.cl del género Campylobacter ${ }^{1}$ mientras que C. lari y C. upsaliensis son las especies de menor frecuencia de aislamiento ${ }^{2}$.

Aunque la Organización Mundial de la Salud recomienda a los países signatarios establecer sistemas de vigilancia epidemiológica respecto de Campylobacter, en Latinoamérica son pocos los países que han avanzado en este sentido ${ }^{3}$. No obstante, en esta región se ha generado información científica sobre este grupo bacteriano con una frecuencia de aislamiento relativamente alta en casos de diarrea, como también en portadores sanos ${ }^{1}$.

En Ecuador existe un Sistema de Vigilancia Epidemiológica (SIVE) y que, a pesar de incluir enfermedades transmitidas por agua y alimentos, la campylobacteriosis no está considerada entre éstas ${ }^{4}$.

Considerando que la información existente sobre Campylobacter en Ecuador es escasa y como una manera de aportar a su conocimiento epidemiológico en este país, el objetivo de esta investigación fue establecer la prevalencia de Campylobacter y su comportamiento in vitro frente a cinco antimicrobianos, en niños con diarrea de la ciudad de Loja.

\section{Metodología}

Se estudiaron 253 muestras fecales obtenidas de niños entre los 7 meses y 9 años de edad, que consultaron por diarrea en dos hospitales de la ciudad de Loja, Ecuador. Las muestras fueron sembradas en agar Butzler e incubadas a $42{ }^{\circ} \mathrm{C}$ en microaerofilia por $48 \mathrm{~h}$ y en agar sangre mediante filtración pasiva, con incubación a $37^{\circ} \mathrm{C}$ en microaerofilia hasta por 5 días 5 .

Las colonias sospechosas fueron identificadas inicialmente por sus características morfológicas (tinción de Gram) y bioquímicas (oxidasa, catalasa, susceptibilidad al ácido nalidíxico y a la cefalotina, e hidrólisis del hipurato y del indoxylacetato). Fueron confirmadas mediante la prueba RPC múltiple propuesta por Yamazaki-Matsune y cols. ${ }^{6}$, la cual fue iniciada con la extracción de ADN de cada cepa mediante el kit E.Z.N.A Tissue ADN (OMEGA bio-tek). Luego, fueron efectuados los ciclos de amplificación para la identificación de género y de las seis especies de Campylobacter que detecta el método (C. hyointestinalis subsp. hyointestinalis, C. coli, C. fetus, C. lari, C. jejuni y C. upsaliensis). Se utilizó como control la cepa $C$. jejuni DSM 4688T.

La susceptibilidad a ampicilina, amoxicilina/ácido clavulánico, eritromicina, ciprofloxacina y gentamicina fue determinada por el método de difusión en disco siguiendo las recomendaciones de EUCAST y del Comité para el Antibiograma de la Sociedad Francesa de Microbiología ${ }^{7}$, teniendo como control la cepa C. jejuni ATCC 33560.

\section{Resultados}

De los 253 niños estudiados, 16 (6,3\%) tuvieron coprocultivo positivo para Campylobacter. Las especies aisladas fueron C. jejuni en 13 casos $(5,1 \%)$ y $C$. coli en $3(1,2 \%)$. No fueron aisladas otras especies del género.

Todas las cepas de C. jejuni y de C. coli aisladas fueron susceptibles a gentamicina y a ampicilina/ácido clavulánico. Frente a ampicilina, las tres cepas de C. coli y 12 (92,3\%) de $C$. jejuni fueron susceptibles. Solamente una cepa $(7,7 \%)$ de $C$. jejuni fue resistente a ampicilina. De las tres cepas de C. coli, una fue resistente y dos susceptibles a eritromicina. En C. jejuni una cepa fue resistente $(7,7 \%)$ y $12(92,3 \%)$ fueron susceptibles. Se encontró alta resistencia a ciprofloxacina, siendo todas las cepas de C. coli y 10 (76,9\%) de $C$. jejuni resistentes a esta fluoroquinolona (Tabla 1 ). No fueron aisladas cepas multirresistentes (resistentes a tres o más antibacterianos) 
Tabla 1. Perfil de susceptibilidad antimicrobiana de Campylobacter jejuni y Campylobacter coli aislados de niños con diarrea de la ciudad de Loja, Ecuador

\begin{tabular}{lcccc}
\hline Antimicrobiano & \multicolumn{2}{c}{$\begin{array}{c}\text { C. jejuni } \\
\mathbf{n = 1 3}\end{array}$} & \multicolumn{2}{c}{$\begin{array}{c}\text { c. coli } \\
\mathbf{n = 3}\end{array}$} \\
Ciprofloxacina & $\mathbf{S}$ & $\mathbf{R}$ & $\mathbf{S}$ & $\mathbf{R}$ \\
\hline Eritromicina & $2(23,1 \%)$ & $10(76,9 \%)$ & 0 & $3(100 \%)$ \\
\hline Gentamicina & $12(92,3 \%)$ & $1(7,7 \%)$ & $2(66,6 \%)$ & $1(33,2 \%)$ \\
Amoxicilina & $13(100 \%)$ & 0 & $3(100 \%)$ & 0 \\
Amoxicillina/ácido clavulánico & $13(100 \%)$ & 0 & $3(100 \%)$ & 0 \\
\hline
\end{tabular}

pero fue encontrada resistencia simultánea a dos antimicrobianos en dos cepas de C.jejuni (ciprofloxacina/eritromicina, ciprofloxacina/ampicilina) y en una de $C$. coli (ciprofloxacina/eritromicina).

\section{Discusión}

Campylobacter jejuni y C. coli son patógenos entéricos reconocidos en todo el mundo y en los países en vías de desarrollo, donde habitualmente no hay vigilancia ni estudio de brotes. La mayoría de los casos son esporádicos ${ }^{1,2}$, como lo fueron los casos incluidos en este estudio. Dieciséis $(6,3 \%)$ de los 253 niños estudiados presentaron coprocultivo positivo para Campylobacter aislándose solamente dos especies del género, C. jejuni $(5,1 \%)$ y $C$. coli $(1,2 \%)$. A pesar de haber sido utilizada la filtración pasiva ${ }^{5}$, no fueron aisladas otras especies de Campylobacter.

La frecuencia de aislamiento encontrada en Loja es menor, aunque cercana, a la informada por Vasco y cols. ${ }^{8}(10 \%)$ en niños del barrio Guamaní en Quito. Sin embargo, ambas son bastante más bajas que $23 \%$ encontrada por Guderian y cols. ${ }^{9}$ en niños de la ciudad de Quito. Es posible que las diferencias con los resultados obtenidos en 1984, se deban a diferencias ambientales de saneamiento básico, las que han debido mejorar desde esa época al presente. Además, también es más baja que las frecuencias informadas por otros estudios de América del Sur $\left(9,1 \%\right.$ en Buenos Aires ${ }^{10}$ y $15,3 \%$ en La Pampa ${ }^{11}$, Argentina; 13,3\% en Lima ${ }^{12}$, Perú; 10,8\% en el Nordeste de Brasi $1^{13}$ y $10 \%$ en el sur de Chile ${ }^{14}$ ). Sin embargo, la proporción de aislamientos de $C$. coli, respecto del total de ambas especies fue de $23,5 \%$, lo que es consistente con la relación C. jejuni/C. coli de 4/1 observada en varios países de América del Sur ${ }^{1}$.

En ninguna de las cepas de $C$. jejuni y de $C$. coli estudiadas se encontró resistencia a gentamicina y a amoxicilina/ácido clavulánico mientras que las tres cepas de C. coli y $12(92,3 \%)$ de $C$. jejuni también fueron susceptibles a ampicilina. Solamente una cepa $(7,7 \%)$ de $C$. jejuni presentó resistencia a ampicilina, pero, a su vez, fue susceptible a amoxicilina/ácido clavulánico, lo que sugiere que esta cepa sería productora de ß-lactamasas, hecho que ha sido observado previamente en otros países latinoamericanos ${ }^{15}$. En relación a eritromicina, solo se encontraron dos cepas resistentes, una de $C$. jejuni y otra de C. coli. Sin embargo, se encontró una alta frecuencia de cepas resistentes a ciprofloxacina, ya que todas las cepas de $C$. coli y $76,9 \%$ de las de $C$. jejuni fueron resistentes a este antimicrobiano. La literatura especializada reconoce que la resistencia a macrólidos y quino- lonas en especies de Campylobacter responde al uso de antibacterianos en el proceso de producción animal y se reflejaría en las cepas aisladas de seres humanos, con mayor predominio de resistencia a quinolonas ${ }^{16,17}$. En esta serie, hubo alta prevalencia de cepas resistentes a ciprofloxacina y una baja resistencia a eritromicina. Estos resultados están en concordancia con estudios previos realizados en esta región donde se encontró una alta resistencia a ciprofloxacina $(62,5-100 \%)$ y una baja o nula resistencia a eritromicina (0-12,5\%) en cepas de Campylobacter aisladas de hígados de pollo para consumo humano ${ }^{18}$ y de materia fecal de pollos de traspatio ${ }^{19}$.

Aunque la gastroenteritis por Campylobacter es generalmente autolimitada, se recomienda el tratamiento antimicrobiano en infecciones sistémicas graves, diarreas prolongadas o en casos de infecciones que ocurren en pacientes de edades extremas, inmunocomprometidos o mujeres embarazadas. Cuando está indicada la terapia antimicrobiana, los macrólidos (eritromicina, claritromicina o azitromicina) son los agentes de elección para el tratamiento de los casos de Campylobacter confirmados por cultivo, especialmente en niños. Las fluoroquinolonas (ciprofloxacina) son utilizadas en adultos para el tratamiento empírico de la diarrea del viajero y casos de diarreas sin agente etiológico diagnosticado mientras que la gentamicina se reserva para el tratamiento de infecciones sistémicas y extraintestinales ${ }^{20,21}$. Considerando el surgimiento de resistencia a los antimicrobianos de elección, la OMS recomienda realizar pruebas de susceptibilidad antimicrobiana in vitro, tanto para proporcionar una orientación sobre el tratamiento apropiado de la campylobacteriosis como para contribuir al conocimiento epidemiológico y a la vigilancia de la resistencia en Campylobacter ${ }^{2}$.

Estos resultados sugieren la necesidad de realizar estudios de mayor alcance en el tiempo, incluyendo cepas de origen humano, ambiental, de animales y de alimentos, para establecer relaciones epidemiológicas en el origen de las cepas de Campylobacter y diseñar estrategias de control y de mitigación de la resistencia en estos agentes zoonóticos de diarrea en el ser humano.

\section{Resumen}

Introducción: Campylobacter es un importante agente de diarrea en el ser humano. En Ecuador, la información sobre Campylobacter es escasa y no existen antecedentes de susceptibilidad antimicrobiana. Objetivo: Describir la prevalencia de Campylobacter en niños con diarrea y su comportamiento in vitro frente a cinco antimicrobianos. Método: Se estudiaron 253 niños entre siete meses y 9 años de edad, que consultaron por diarrea en dos hospitales de la ciudad de Loja. Se realizó cultivo de muestras fecales e identificación por pruebas fenotípicas y por RPC múltiple. La susceptibilidad antimicrobiana fue determinada por el método de difusión en disco. Resultados: Campylobacter fue diagnosticado en 16 $(6,3 \%)$ de las muestras, aislándose $C$. jejuni en $13(5,1 \%)$ y $C$. coli en 3 $(1,2 \%)$. Todas las cepas fueron susceptibles a gentamicina y ampicilina/ ácido clavulánico, con baja resistencia a ampicilina y eritromicina y alta resistencia a ciprofloxacina.

\section{Referencias bibliográficas}

1.- Fernández H. Campylobacter y campylobacteriosis: una mirada desde América del Sur. Rev Peru Med Exp Salud Pública 2011; 28: 121-27.

2.- World Health Organization. 2013. The global view of campylobacteriosis: 
report of an expert consultation, Utrecht, Netherlands, 9-11 July 2012. WHO Document Production Services, Geneva, Switzerland. pp 57.

3.- Instituto de Salud Pública de Chile. Vigilancia de laboratorio de Campylobacter spp., 2005-2013. Bol Inst Salud Pública Chile 2014; 4: 1-17.

4.- Ministerio de Salud Pública del Ecuador. Enfermedades transmitidas por agua y alimentos. Gaceta Epidemiológica 2017. SE-23: 9-12. Disponible en: http://www.salud.gob.ec/wp-content/uploads/2013/02/Gaceta-GeneralSE-23.pdf

5.- Fernández H, Vera F, Villanueva MP, García A. Occurrence of Campylobacter species in healthy well-nourished and malnourished children. Braz J Microbiol 2008; 39: 56-8.

6.- Yamazaki-Matsune W, Taguchi M, Seto K, Kawahara R, Kawatsu K, Kumeda Y, et al. Development of a multiplex PCR assay for identification of Campylobacter coli, Campylobacter fetus, Campylobacter hyointestinalis subsp. hyointestinalis, Campylobacter jejuni, Campylobacter lari and Campylobacter upsaliensis. J Med Microbiol 2007; 56: 1467-73.

7.- Société Française de Microbiologie. Comité de l'AntibiogrammeRecommandations 2014.2014. Société Française de Microbiologie, Paris,France, pp 1-115.

8.- Vasco G, Trueba G, Atherton R, Calvopiña M, Cevallos W, Andrade T, et al. Identifying etiological agents causing diarrhea in low income Ecuadorian communities. Am J Trop Med Hyg 2014; 91: 563-9. doi: 10.4269/ ajtmh.13-0744.

9.- Guderian R H, Ordóñez G, Bossano R. Acute diarrhea associated with Campylobacter and other pathogens in Quito, Ecuador. Bol Oficina Sanit Panam 1987; 102: 333-9.

10.- López C, Agostini A, Giacoboni G, Cornero F, Tellechea D, Trinidad J J. Campilobacteriosis en una comunidad de bajos recursos de Buenos Aires, Argentina. Rev Sci Tech Off Int Epiz 2003; 22: 1013-20.

11.- Tamborini A L, Casabona L M, Viñas M R, Asato V, Hoffer A, Farace M I, et al. Campylobacter spp.: prevalencia y caracterización feno-genotípica de aislamientos de pacientes con diarrea y de sus mascotas en la provincia de La Pampa, Argentina. Rev Argent Microbiol 2012; 44: 266-71.

12.- Perales M, Camiña M, Quioñes C. Infección por Campylobacter y Shigella como causa de diarrea aguda infecciosa en niños menores de dos años en el distrito de la Victoria, Lima-Perú. Rev Peru Med Exp Salud Pública
2002;19: 186-92.

13.- Quetz JDS, Lima IFN, Havt A, De Carvalho EB, Lima NL, Soares AM, et al. Campylobacter jejuni and Campylobacter coli in children from communities in Northeastern Brazil: molecular detection and relation to nutritional status. Diagn Microbiol Infect Dis 2010; 67: 220-7. doi: 10.1016/j.diagmicrobio.2010.02.025

14.- Collado L, Gutiérrez M, González M, Fernández H. Assessment of the prevalence and diversity of emergent campylobacteria in human stool samples using a combination of traditional and molecular methods. Diagn Microbiol Infect Dis 2013; 75: 434-6. doi: 10.1016/j. diagmicrobio.2012.12.006.

15.- Fernández H, Oval A. Occurrence of Campylobacter jejuni and Campylobacter coli biotypes and antimicrobial susceptibility in healthy dogs in Southern Chile. Acta Scient Vet 2013; 41: 1100.

16.- Gibreel A, Taylor D E. Macrolide resistance in Campylobacter jejuni and Campylobacter coli. J Antimicrob Chemother 2006; 58: 243-55.

17.- Pollett S, Rocha C, Zerpa R, Patiño L, Valencia A, Camiña M, et al. Campylobacter antimicrobial resistance in Peru: a ten-year observational study. BMC Infect Dis 2012; 12: 193. doi: 10.1186/1471-2334-12-193.

18.- Simaluiza R J, Toledo Z, Ochoa S, Fernández H. The prevalence and antimicrobial resistance of Campylobacter jejuni and Campylobacter coli in chicken livers used for human consumption in Ecuador. J Anim Vet Adv 2015; 14: 6-9.

19.- Ochoa S, Simaluiza R J, Toledo Z, Fernández H. Frequency and antimicrobial behavior of thermophilic Campylobacter species isolated from Ecuadorian backyard chickens. Arch Med Vet 2016; 48: 311-4.

20.- Fernández H, Pérez-Pérez G. Campylobacter: fluoroquinolone resistance in Latin-American countries. Arch Med Vet 2016; 48: 255-9.

21.- Lapierre L, Arias M L, Fernández H. Chapter 10: Antimicrobial resistance in Campylobacter spp. Fonseca B, Fernández H, Rossi D, editors. Campylobacter spp. and Related Organisms in Poultry. Pathogen-Host Interactions, Diagnosis and Epidemiology. Springer International Publishing Switzerland; 2016, p. 165-83. 